\title{
CCMS technology enables improved proteomic analysis through functional isolation of subproteomes
}

\section{Capture Compound mass spectrometry (CCMS) is a technology to reduce the complexity of biological samples through selective isolation of targeted proteins. caprotec's Capture Compounds ${ }^{\mathrm{TM}}$ are small, synthetic molecules that can be used to interrogate native proteins, even lipophilic membrane proteins. CCMS allows discovering, isolating and profiling members of functional protein families within a variety of biological samples.}

The proteome is a complex mixture of proteins and peptides in varying concentrations and conditions. Analyzing the structure and function of all cellular proteins with the goal of understanding the interplay of the different pathways is a challenging task even with today's advanced separation and analysis capabilities. Capture Compounds provide a new tool set for functional protein separation.

There are various methods for analyzing complex protein mixtures ${ }^{1,2}$, including two-dimensional gel electrophoresis ${ }^{3}$, affinity chromatography ${ }^{4}$ and mass spectrometry. These methods are very useful tools but also have limitations-for example, in the analysis of lipophilic or very large proteins or in the investigation of lowaffinity small molecule-protein interactions.

caprotec $^{\mathrm{TM}}$ bioanalytics has developed an innovative technology to substantially reduce the complexity of biological samples through selective and functional isolation of targeted protein via small, multifunctional molecules, called Capture Compounds (Fig. 1).

Capture Compounds allow a reversible affinity interaction between their 'selectivity function' and specific proteins. A 'reactivity function' forms a covalent bond with the interacting proteins, and a 'sorting function' enables the isolation directly from cell lysates.

The Capture Compound mass spectrometry (CCMS) technology can use protein samples from cell culture, bacteria, plants or tissues, without the need for depletion of albumin or other highly abundant proteins. As the assay is not dependent on substrate conversion, target molecules do not need to be active enzymes.

The interacting target proteins are isolated in three steps (Fig. 2). In the first step, Capture Compounds are incubated with a biologi-

\section{Erik Dülsner, Thomas Lenz \& Christian Jurinke}

caprotec bioanalytics $\mathrm{GmbH}$, Volmerstrasse 5, 12489 Berlin, Germany.

Correspondence should be addressed to E.D. (erik.duelsner@caprotec.com) or

C.J. (christian.jurinke@caprotec.com).

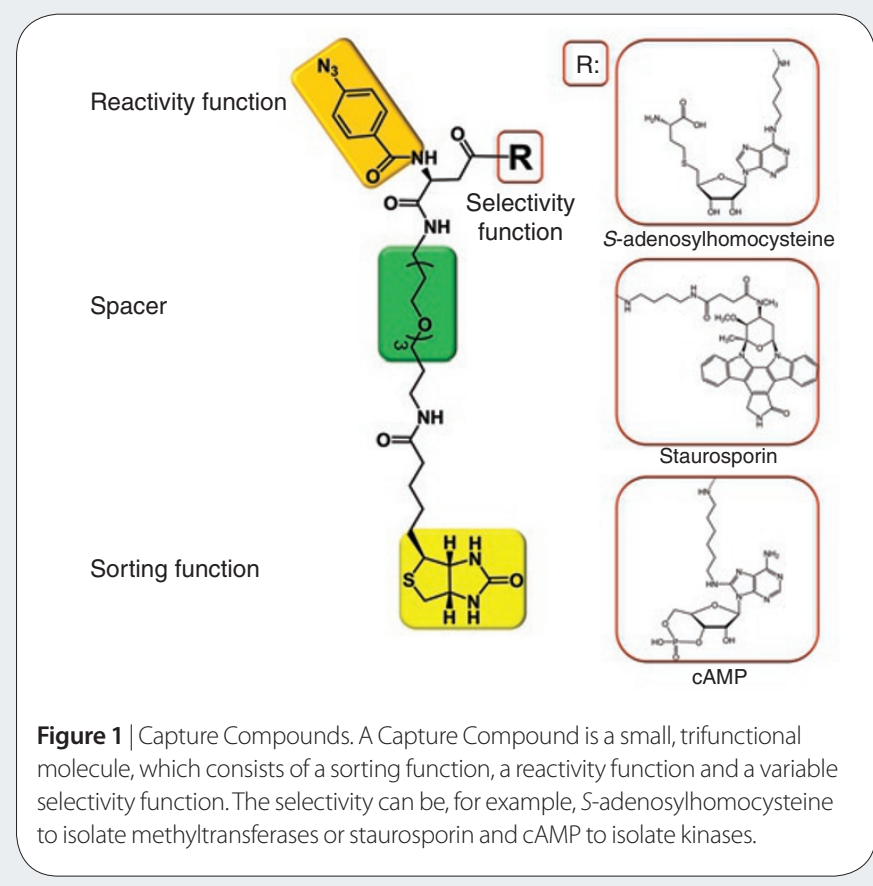

cal sample to allow a reversible, affinity-driven interaction with the targets. In the second step, the reactivity function is activated using UV light and covalently attached to the proteins using caprotec's caproBox $^{\mathrm{TM}}$. This instrument combines timed UV irradiation and temperature control in one system and allows a convenient reproducible photo-cross-linking. In the third step, the sorting function is used to isolate captured proteins from the mixture by means of streptavidin-coated magnetic beads. The isolated proteins can be characterized by gel electrophoresis or mass spectrometry.

One important advantage of the CCMS technology is that capture reactions are performed in a simple, homogeneous protocol that is amenable to automation and scaling up with off-the-shelf liquid handling devices. 


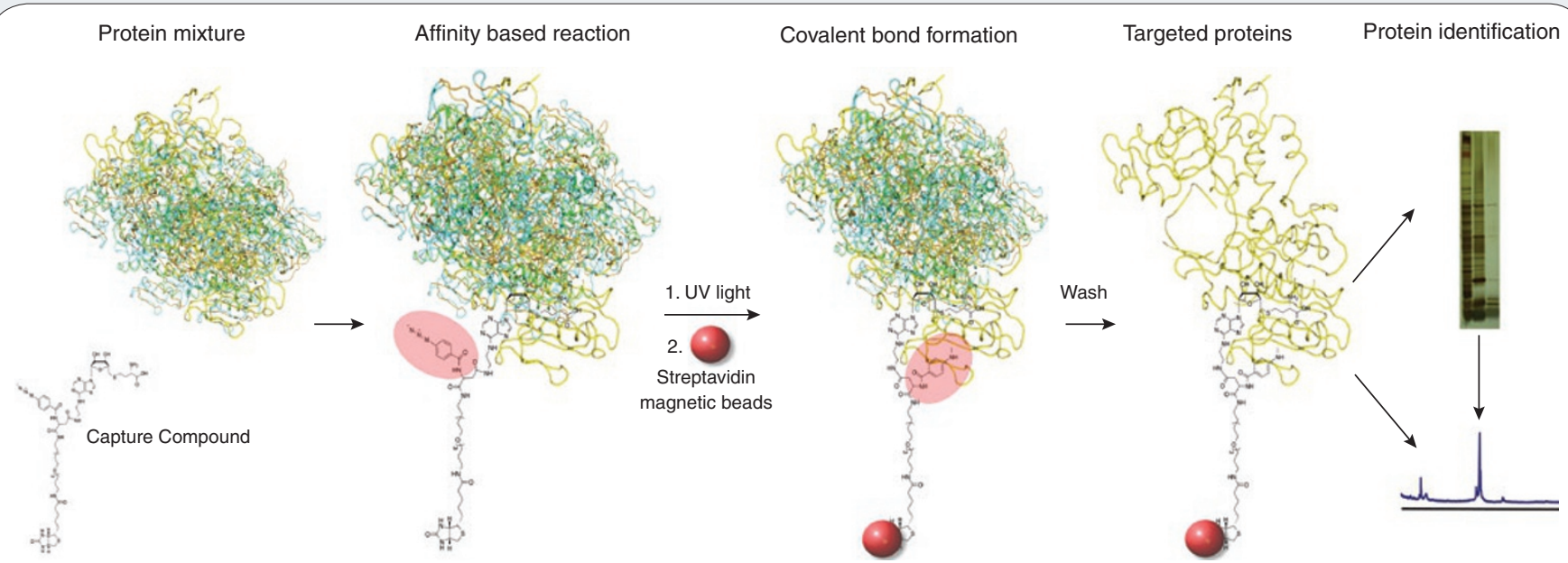

Figure 2 | Capture Compounds bind proteins through reversible affinity interaction. A covalent bond between Capture Compound and target protein is generated by photo-cross-linking. Streptavidin-biotin interactions are used to isolate captured proteins for further analyses. CCMS experiments can be performed within a working day.

The data generated in CCMS experiments provide a wide range of information-for example, mass spectrometry can identify protein interaction partners or post-translational modifications.

Another advantage of CCMS is the possibility of incorporating internal controls into the experiment. For example, the functional selectivity of the capture event can be monitored through the addition of competitors in a control reaction.

During the CCMS process, the equilibrium between the selectivity function and interacting proteins is practically frozen in the UV-light cross-linking step. This feature can be used to obtain quantitative information about dissociation constant $\left(K_{\mathrm{d}}\right)$ values between the ligand and the affinity-selected proteins (see http://www.caprotec.com/).

Analyzing the structure and function of proteins is the major goal of

(n)
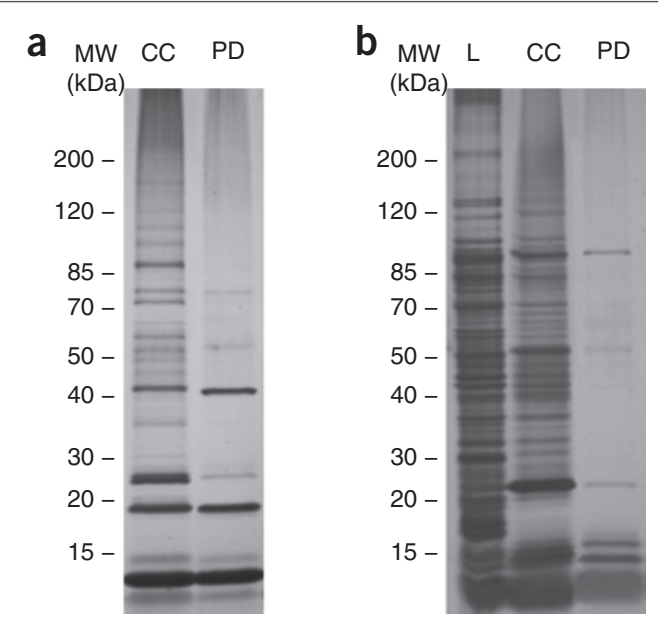

Figure $\mathbf{3}$ | Capturing reduces the complexity of the biological samples. (a,b) SDS-PAGE separation followed by silver staining of SAM-binding proteins (a) and CAMP-binding proteins (b) from Escherichia coli cell lysates $(\mathrm{L})$ isolated using Capture Compounds (CC) and in a pull-down assay (PD). Isolation using Capture Compounds yields more captured proteins than does a pull-down assay. The selectivity of either the capture event or pull-down assay can be directly monitored through addition of competitors in a control experiment. understanding the different signaling pathways. Pull-down assays are widely used to enrich the proteins of interest. The basis of pull-down assays is the affinity between a selectivity function (for example, an antibody) and the targeted protein. However, proteins with low affinities are frequently lost in washing steps. The CCMS process uses a covalent cross-link to the targeted protein. This covalent attachment allows the analysis of low-affinity interactions because less material is lost during washing steps. CCMS provides a superior yield of targeted proteins when compared to pull-down assays (Fig. 3). Another advantage of CCMS technology is the gel-free characterization of captured proteins directly through liquid chromatography-mass spectrometry.

In conclusion, using Capture Compounds, we demonstrated a considerable reduction of complexity in biological samples. Use of Capture Compounds allows the forced enrichment of affinity-interacting proteins at a much higher efficiency than single pull-down assays. This advantage is achieved by a covalent cross-link between Capture Compounds and the targeted protein. With its high reproducibility and efficiency, the CCMS technology is poised to play an important role in analyzing the structure and function of cellular proteins with the goal of understanding the time-dependent interplay of the different biochemical and signaling pathways.

Capture Compounds are available as caproKits ${ }^{\mathrm{TM}}$ from caprotec bioanalytics. Currently available caproKits target kinases, methyltransferases and cyclicAMP (cAMP)-binding proteins.

1. Aebersold, R. \& Mann, M. Mass spectrometry-based proteomics. Nature $\mathbf{4 2 2}$, 198-207 (2003).

2. Patterson, S.D. \& Aebersold, R. Proteomics: the first decade and beyond. Nat. Genet 33, 311-323 (2003).

3. Lilley, K.S., Razzaq, A. \& Dupree, P. Two-dimensional gel electrophoresis: recent advances in sample preparation, detection and quantitation. Curr. Opin. Chem. Biol. 6, 46-50 (2002).

4. Heftmann, E. Affinity chromatography. J. Biochem. Biophys. Methods (Special issue) 49, 1-750 (2001)

This article was submitted to Nature Methods by a commercial organization and has not been peer reviewed. Nature Methods takes no responsibility for the accuracy or otherwise of the information provided. 